\title{
Necessary and sufficient conditions for uniform stability of Volterra integro-dynamic equations using new resolvent equation
}

\author{
Murat Adivar and Youssef N. Raffoul
}

\begin{abstract}
We consider the system of Volterra integro-dynamic equations

$$
x^{\Delta}(t)=A(t) x(t)+\int_{t_{0}}^{t} B(t, s) x(s) \Delta s
$$

and obtain necessary and sufficient conditions for the uniform stability of the zero solution employing the resolvent equation coupled with the variation of parameters formula. The resolvent equation that we use for the study of stability will have to be developed since it is unknown for time scales. At the end of the paper, we furnish an example in which we deploy an appropriate Lyapunov functional. In addition to generalization, the results of this paper provides improvements for its counterparts in integro-differential and integro-difference equations which are the most important particular cases of our equation.
\end{abstract}

\section{Introduction}

In this paper we assume the reader is familiar with the basic calculus of time scales. Hereafter, we denote by $\mathbb{T}$ and $[a, b]_{\mathbb{T}}$ a time scale (a nonempty closed

Key Words: Lyapunov functional, New resolvent equation, Time scales, Uniform stability, Volterra.

2010 Mathematics Subject Classification: Primary: 34K20, 34N05; Secondary: 45J05, $45 \mathrm{D} 05$.

Received: April, 2012.

Revised: May, 2012.

Accepted: February, 2013. 
subset of reals) and a time scale interval defined by $[a, b]_{\mathbb{T}}=[a, b] \cap \mathbb{T}$, respectively. The intervals, $(a, b)_{\mathbb{T}},[a, b)_{\mathbb{T}}$, and $(a, b]_{\mathbb{T}}$ are defined similarly. For a comprehensive review on time scales we refer to [6] and [7].

In [2], we have developed various type of results concerning resolvent and variation of parameters of integral dynamic equations and Volterra integrodynamic equations on time scales. In particular, the authors in [2] considered the scalar integral dynamic equation

$$
x(t)=f(t)+\int_{t_{0}}^{t} a(t, s) x(s) \Delta s, t \in\left[t_{0}, \infty\right)_{\mathbb{T}}
$$

and showed the existence of the resolvent equation equation $r(t, s)$ of (1) that satisfies

$$
r(t, s)=-a(t, s)+\int_{\sigma(t)}^{t} r(t, u) a(u, s) \Delta u .
$$

Then using the resolvent given by (2) they obtained the variation of parameters formula

$$
x(t)=f(t)-\int_{t_{0}}^{t} r(t, u) f(u) \Delta u .
$$

As a consequence, they showed if $r(t, s)$ satisfies (2) and that $f(t)$ is bounded, then every solution $x(t)$ of $(1)$ is bounded if and only if

$$
\sup _{t \in\left[t_{0}, T\right]_{\mathbb{T}}} \int_{t_{0}}^{t}|r(t, s)| \Delta s<\infty, T>t_{0},
$$

holds. In [1] the first author introduced the principal matrix solution $Z(t, s)$ of the linear Volterra vector integro-dynamic equation

$$
x^{\Delta}(t)=A(t) x(t)+\int_{s}^{t} B(t, u) x(u) \Delta u
$$

and proved that it is the unique matrix solution of

$$
Z^{\Delta_{t}}(t, s)=A(t) Z(t, s)+\int_{s}^{t} B(t, u) Z(u, s) \Delta u, \quad Z(s, s)=I .
$$

He also showed that the solution of

$$
x^{\Delta}(t)=A(t) x(t)+\int_{s}^{t} B(t, u) x(u) \Delta u+f(t), \quad x(\tau)=x_{0}
$$

is unique and given by the variation of parameters formula

$$
x(t)=Z(t, \tau) x_{0}+\int_{\tau}^{t} Z(t, \sigma(s)) f(s) \Delta s,
$$


where $\sigma: \mathbb{T} \rightarrow \mathbb{T}$ is the forward jump operator defined by $\sigma(t):=\inf \{s \in \mathbb{T}$ : $s>t\}$. Qualitative and quantitative properties of Volterra integral equation have been studied in [4], [5], [10], and [13].

In this paper we consider the system of Volterra integro-dynamic equations on time scale of the form

$$
x^{\Delta}(t)=A(t) x(t)+\int_{t_{0}}^{t} B(t, s) x(s) \Delta s, \quad t \in\left[t_{0}, \infty\right)_{\mathbb{T}},
$$

where $A$ is an $n \times n$ matrix function that is continuous on $\left[t_{0}, \infty\right)_{\mathbb{T}}, B$ is an $n \times n$ matrix function that is continuous on

$$
\Omega:=\left\{(t, u) \in \mathbb{T} \times \mathbb{T}: t_{0} \leq u \leq t<\infty\right\} .
$$

We will develop a companion resolvent equation to (4) and make use of it to obtain a variation of parameters formula on all possible solutions of (5). As a consequence, we provide necessary and sufficient conditions for the uniform stability of the zero solution of (5). Toward the end of the paper, (i.e., in Remarks 5.4 and 5.5), we compare our results to known results in special cases of time scales and easily show that this paper provides improvement over those results.

\section{The Adjoint Equation}

In this section, we make use of the adjoint equation of (5) to show that the matrix solution $Z(t, s)$ of (5), Adivar proved its existence in [1], is equivalent to our resolvent $R(t, s)$. That would put the existence of $R(t, s)$ to rest. We begin by making the first definition.

Definition 2.1. The adjoint to (5) is

$$
y^{\Delta_{s}}(s)=-A^{T}(s) y(\sigma(s))-\int_{\sigma(s)}^{t} B^{T}(u, s) y(\sigma(u)) \Delta u, s \in\left[t_{0}, t\right]_{\mathbb{T}} .
$$

The next theorem establishes the existence and uniqueness of solutions of (6).

Theorem 2.2. For a fixed $t \in\left[t_{0}, \infty\right)_{\mathbb{T}}$ and a given $y_{0} \in \mathbb{R}^{n}$, there is a unique solution $y(s)$ of $(6)$ on $\left[t_{0}, t\right]_{\mathbb{T}}$ with $y(t)=y_{0}$.

Proof. Integrate (6) from $s$ to $t$ and replace $y(t)$ with $y_{0}$ to obtain

$$
P \phi(t):=y_{0}+\int_{s}^{t}\left[A^{T}(u)+\int_{\sigma(u)}^{t} B^{T}(u, v) \Delta v\right] \phi(\sigma(u)) \Delta u
$$


for all $\phi \in C_{y_{0}}[s, T]_{\mathbb{T}}$, where $\phi \in C_{y_{0}}[s, T]_{\mathbb{T}}$ is defined in [1]. The rest of the proof is identical to that of Lemma 7 and Lemma 8 of [1] and hence we omit.

Definition 2.3. The principal matrix solution of (6) is the $n \times n$ matrix function given by

$$
Q(t, s)=\left[\begin{array}{lll}
y^{1}(t, s) & y^{2}(t, s) \ldots y^{n}(t, s)
\end{array}\right],
$$

where $y^{i}(t, s)$ is the unique solution of $(6)$ on $\left[t_{0}, t\right]_{\mathbb{T}}$ that satisfies condition $y^{i}(t, t)=e^{i}, i=1,2, \ldots, n$, where $\left\{e^{1}, e^{2}, \ldots, e^{n}\right\}$ is the standard basis for $\mathbb{R}^{n}$.

We remark that Definition 2.3 implies that $Q(t, s)$ is the unique matrix solution of

$$
\begin{gathered}
Q^{\Delta_{s}}(t, s)=-A^{T}(s) Q(t, \sigma(s))-\int_{\sigma(s)}^{t} B^{T}(u, s) Q(t, \sigma(u)) \Delta u, \quad s \in\left[t_{0}, t\right]_{\mathbb{T}} \\
\text { and } Q(t, t)=I .
\end{gathered}
$$

By a similar argument as in [1, Theorem 12], we conclude that for a given $y_{0} \in \mathbb{R}^{n}$ the unique solution of (6) satisfying $y(t)=y_{0}$ is

$$
y(s)=Q(t, s) y_{0}, \quad s \in\left[t_{0}, t\right]_{\mathbb{T}} .
$$

Let $r(s)=y^{T}(s)$ and take the transpose in (6) to obtain

$$
r^{\Delta_{s}}(s)=-r(\sigma(s)) A(s)-\int_{\sigma(s)}^{t} r(\sigma(u)) B(u, s) \Delta u, \quad s \in\left[t_{0}, t\right]_{\mathbb{T}} .
$$

The solution satisfying the condition $r(t)=y_{0}^{T}=: r_{0}$ is the transpose of (7) namely

$$
y^{T}(s)=y_{0}^{T} Q^{T}(t, s), \quad s \in\left[t_{0}, t\right]_{\mathbb{T}}
$$

or

$$
r(s)=r_{0} R(t, s)=Q^{T}(t, s) .
$$

Thus, $R(t, s)$ is the principal matrix solution of the transposed equation. As a result, Lemma 18 of [1] has the following adjoint counterpart.

Lemma 2.4. The solution of (8) on $\left[t_{0}, t\right]_{\mathbb{T}}$ satisfying the condition $r(t)=r_{0}$ is

$$
r(s)=r_{0} R(t, s),
$$

where $R(t, s)$ is the principal matrix solution of (8). 


\section{New Resolvent Equation}

Before we move to obtaining stability results, we will show the existence of the resolvent $R(t, s)$ of (5). We will use the results of the previous section and show that the principal matrix $Z(t, s)$ of $(5)$ is equivalent to $R(t, s)$ where $R(t, s)$ will satisfy a different equation. Once we show the equivalency, we would have established the existence of $Z(t, s)$ which was done in [1].

Theorem 3.1. $R(t, s)$ is equivalent to $Z(t, s)$.

Proof. Let $s \in\left[t_{0}, t\right]_{\mathbb{T}}$ and let $r(s)$ be the unique solution of $(8)$ with $r(t)=r_{0}$. Consider differentiation of the product

$$
[r(u) Z(u, s)]^{\Delta_{u}}=r^{\Delta_{u}}(u) Z(u, s)+r(\sigma(u)) Z^{\Delta_{u}}(u, s) .
$$

An integration of (12) from $s$ to $t$ gives

$$
r_{0} Z(t, s)-r(s)=\int_{s}^{t}\left[r(\sigma(u)) Z^{\Delta_{u}}(u, s)+r^{\Delta_{u}}(u) Z(u, s)\right] \Delta u .
$$

Substituting $r^{\Delta_{u}}(u)$ that is given by (8) into (13), we obtain

$$
\begin{aligned}
r_{0} Z(t, s)-r(s) & =\int_{s}^{t} r(\sigma(u))\left[Z^{\Delta_{u}}(u, s)-A(u) Z(u, s)\right] \Delta u \\
& -\int_{s}^{t}\left[\int_{\sigma(u)}^{t} r(\sigma(v)) B(v, u) \Delta v\right] Z(u, s) \Delta u .
\end{aligned}
$$

A simple change of order of integration yields to

$$
\begin{aligned}
\int_{s}^{t}\left[\int_{\sigma(u)}^{t} r(\sigma(v)) B(v, u) \Delta v\right] Z(u, s) \Delta u & =\int_{s}^{t} r(\sigma(v))\left[\int_{s}^{v} B(v, u) Z(u, s) \Delta u\right] \Delta v \\
& =\int_{s}^{t} r(\sigma(u))\left[\int_{s}^{u} B(u, v) Z(v, s) \Delta v\right] \Delta u .
\end{aligned}
$$

With this in mind, the above expression becomes

$r_{0} Z(t, s)-r(s)=\int_{s}^{t}\left[r(\sigma(u))\left[Z^{\Delta_{u}}(u, s)-A(u) Z(u, s)-\int_{s}^{u} B(u, v) Z(v, s) \Delta v\right] \Delta u\right.$.

By (4) the integral is zero, which leaves us with

$$
r_{0} R(t, s)=r(s)=r_{0} Z(t, s) .
$$


Now we are ready to make the following definition.

Definition 3.2. The resolvent matrix solution $R(t, s)$ of $(5)$ is the unique solution of

$$
R^{\Delta_{s}}(t, s)=-R(t, \sigma(s)) A(s)-\int_{\sigma(s)}^{t} R(t, \sigma(u)) B(u, s) \Delta u, \quad R(t, t)=I
$$

where $I$ is the $n \times n$ identity matrix.

Our variation of parameters formula depends on an initial function $\varphi$ and therefore we state the following definition.

Definition 3.3. Let $\varphi(t)$ be a given bounded and initial function. We say $x\left(t, \tau_{0}, \varphi\right)$ is a solution of (5) if $x(t)=\varphi(t)$ for $t_{0} \leq t \leq \tau_{0}$ and $x\left(t, \tau_{0}, \varphi\right)$ satisfies (5) for $t \geq \tau_{0}$.

Theorem 3.4. Let $\varphi$ be a given bounded and continuous initial function defined on $t_{0} \leq t \leq \tau_{0} . x(t)$ is a solution of (5) if and only if

$$
x(t)=R\left(t, \tau_{0}\right) \varphi\left(\tau_{0}\right)+\int_{\tau_{0}}^{t} R(t, \sigma(s)) \int_{t_{0}}^{\tau_{0}} B(s, u) \varphi(u) \Delta u \Delta s .
$$

Proof. Note that

$$
[R(t, s) x(s)]^{\Delta}=R(t, \sigma(s)) x^{\Delta}(s)+R^{\Delta_{s}}(t, s) x(s) .
$$

An integration of (16) from $\tau_{0}$ to $t$ gives

$$
R(t, t) x(t)-R\left(t, \tau_{0}\right) \varphi\left(\tau_{0}\right)=\int_{\tau_{0}}^{t}\left[R(t, \sigma(s)) x^{\Delta}(s)+R^{\Delta_{s}}(t, s) x(s)\right] \Delta s .
$$

Hence, (17) implies that

$$
x(t)=R\left(t, \tau_{0}\right) \varphi\left(\tau_{0}\right)+\int_{\tau_{0}}^{t}\left[R(t, \sigma(u)) x^{\Delta}(u)+R^{\Delta_{u}}(t, u) x(u)\right] \Delta u .
$$

Using (5) into (18) yields

$$
\begin{aligned}
x(t) & =R\left(t, \tau_{0}\right) \varphi\left(\tau_{0}\right)+\int_{\tau_{0}}^{t} R(t, \sigma(u)) A(u) x(u) \Delta u \\
& +\int_{\tau_{0}}^{t} R(t, \sigma(u)) \int_{t_{0}}^{u} B(u, s) x(s) \Delta s \Delta u+\int_{\tau_{0}}^{t} R^{\Delta_{u}}(t, u) x(u) \Delta u .
\end{aligned}
$$


Next we consider the third term on the right side of (19). That is

$$
\begin{aligned}
\int_{\tau_{0}}^{t} R(t, \sigma(u)) \int_{t_{0}}^{u} B(u, s) x(u) \Delta s \Delta u & =\int_{\tau_{0}}^{t} R(t, \sigma(u)) \int_{t_{0}}^{\tau_{0}} B(u, s) \varphi(s) \Delta s \Delta u \\
& +\int_{\tau_{0}}^{t} R(t, \sigma(u)) \int_{\tau_{0}}^{u} B(u, s) x(s) \Delta s \Delta u .
\end{aligned}
$$

By changing the limits of integration see [1] we get

$$
\int_{\tau_{0}}^{t} R(t, \sigma(u)) \int_{\tau_{0}}^{u} B(u, s) x(s) \Delta s \Delta u=\int_{\tau_{0}}^{t}\left\{\int_{\sigma(s)}^{t} R(t, \sigma(u)) B(u, s) \Delta u\right\} x(s) \Delta s .
$$

With all this in mind, equation (19) implies that

$$
\begin{aligned}
x(t) & =R\left(t, \tau_{0}\right) \varphi\left(\tau_{0}\right)+\int_{\tau_{0}}^{t} R(t, \sigma(u)) \int_{t_{0}}^{\tau_{0}} B(u, s) \varphi(s) \Delta s \Delta u \\
& +\int_{\tau_{0}}^{t}\left[R(t, \sigma(s)) A(s)+\int_{\sigma(s)}^{t} R(t, \sigma(u)) B(u, s) \Delta u+R^{\Delta_{s}}(t, s)\right] x(s) \Delta s .
\end{aligned}
$$

Now the third term on the right is zero due to (14). Interchange $s$ with $u$ to get (15)

\section{$4 \quad$ Stability}

For $x \in \mathbb{R}^{n},|x|$ denotes the Euclidean norm of $x$. For any $n \times n$ matrix $A$, define the norm of $A$ by $|A|=\sup \{|A x|:|x| \leq 1\} . C(X, Y)$ denotes the set of continuous functions $\phi: X \rightarrow Y$. Let $C(t)$ denote the set of continuous functions $\phi:\left[t_{0}, t\right]_{\mathbb{T}} \rightarrow \mathbb{R}^{n}$ and $\|\phi\|=\sup \left\{|\phi(s)|: t_{0} \leq s \leq t\right\}$. For each $\tau_{t_{0}} \in\left[t_{0}, t\right]_{\mathbb{T}}$ and $\phi \in C\left(\tau_{0}\right)$, there is a unique function $x:\left[t_{0}, t\right]_{\mathbb{T}} \rightarrow \mathbb{R}^{n}$ which satisfies $(5)$ on $\left[\tau_{0}, \infty\right)_{\mathbb{T}}$ with $x(s)=\phi(s)$ for $s \in\left[t_{0}, \tau_{0}\right]_{\mathbb{T}}$. Such a function $x(t)$ is called a solution of $(5)$ through $\left(\tau_{0}, \phi\right)$ and is denoted by $x\left(t, \tau_{0}, \phi\right)$.

Definition 4.1. The zero solution of (5) is stable if for each $\varepsilon>0$ and each $\tau_{0} \geq t_{0}$, there exists a $\delta=\delta\left(\varepsilon, \tau_{0}\right)>0$ such that $\left[\phi \in C\left(\tau_{0}\right),:\|\phi\|<\delta\right]$ imply $\left|x\left(t, \tau_{0}, \phi\right)\right|<\varepsilon$ for all $t \geq \tau_{0}$. The zero solution of (5) is uniformly stable (US) if $\delta$ is independent of $\tau_{0}$.

The following theorem is an extension of Theorem 1 of Miller's result, [11] on uniform stability. 
Theorem 4.2. The zero solution of (5) is uniformly stable if and only if

$$
\sup _{t \in\left[t_{0}, \infty\right)_{\mathbb{T}}}\left\{\left|R\left(t, \tau_{0}\right)\right|+\int_{t_{0}}^{\tau_{0}}\left|\int_{\tau_{0}}^{t} R(t, \sigma(s)) B(s, u) \Delta s\right| \Delta u\right\}<+\infty .
$$

Proof. By (20) there exists a constant $E>0$ such that

$$
\sup _{t \in\left[t_{0}, \infty\right)_{\mathbb{T}}}\left\{\left|R\left(t, \tau_{0}\right)\right|+\int_{t_{0}}^{\tau_{0}}\left|\int_{\tau_{0}}^{t} R(t, \sigma(s)) B(s, u) \Delta s\right| \Delta u\right\}<E .
$$

By interchanging the order of integration in (15) we get

$$
\begin{aligned}
|x(t)| & \leq\left\{\left|R\left(t, \tau_{0}\right)\right|+\int_{t_{0}}^{\tau_{0}}\left|\int_{\tau_{0}}^{t} R(t, \sigma(s)) B(s, u) \Delta s\right| \Delta u\right\}\|\phi\| \\
& \leq E\|\phi\| .
\end{aligned}
$$

This proves that the zero solution of (5) is US.

Conversely, suppose that the zero solution of (5) is US. Then for $\varepsilon=1$, there exists $\delta>0$ such that $\left[\tau_{0} \geq t_{0}, \phi \in C\left(\tau_{0}\right),\|\phi\|<\delta, t \geq \tau_{0}\right]$ imply $\left|x\left(t, \tau_{0}, \phi\right)\right|<1$. For every positive integer $m$ define

$$
\tau_{m}:=\min \left[\tau_{0}-\frac{1}{m}, \tau_{0}\right]_{\mathbb{T}}
$$

and

$$
\phi_{m}(s):=\left\{\begin{array}{lll}
m\left(s-\tau_{0}+\frac{1}{m}\right) \omega & \text { for } & s \in\left[\tau_{m}, \tau_{0}\right]_{\mathbb{T}} \\
0 & \text { for } & s \in\left[t_{0}, \tau_{0}-\frac{1}{m}\right]_{\mathbb{T}}
\end{array},\right.
$$

where $\omega$ is the $n \times 1$ unit vector. It is clear from (22) that $\phi_{m}\left(\tau_{0}\right)=\omega$ and $\left|\phi_{m}(s)\right| \leq 1$ for all $s \in\left[t_{0}, \tau_{0}\right]_{\mathbb{T}}$. If we let $\psi_{m}(s)=\frac{\delta}{2} \phi_{m}(s)$, then $\left|\psi_{m}(s)\right| \leq \frac{\delta}{2}$. Hence, we have $\left|x\left(t, \tau_{0}, \psi_{m}(s)\right)\right| \leq 1$. Thus from the variation of parameters formula given by (15) we have,

$$
x\left(t, \tau_{0}, \psi_{m}\right)=R\left(t, \tau_{0}\right) \psi_{m}\left(\tau_{0}\right)+\int_{\tau_{0}}^{t} R(t, \sigma(s)) \int_{t_{0}}^{\tau_{0}} B(s, u) \psi_{m}(u) \Delta u \Delta s
$$

which implies that

$$
\left|R\left(t, \tau_{0}\right)\right| \frac{\delta}{2} \leq 1+\frac{\delta}{2} \int_{\tau_{0}}^{t}|R(t, \sigma(s))|\left|\int_{\tau_{m}}^{\tau_{0}} B(s, u) \phi_{m}(u) \Delta u\right| \Delta s
$$

Since $\tau_{m} \rightarrow \tau_{0}$ we have

$$
\int_{\tau_{0}}^{t}|R(t, \sigma(s))|\left|\int_{\tau_{m}}^{\tau_{0}} B(s, u) \phi_{m}(u) d u\right| d s \rightarrow 0
$$


as $m \rightarrow \infty$. Thus, (24) gives

$$
\left|R\left(t, \tau_{0}\right)\right| \leq \frac{2}{\delta} \text { for all } t \in\left[t_{0}, \infty\right)_{\mathbb{T}} .
$$

Next, let $\phi \in C\left(\tau_{0}\right)$ with $\|\phi\|<1$ and define $\bar{\phi}=\delta \phi$. Then $\|\bar{\phi}\|<\delta$. By the definition of $\delta$, we have $\left|x\left(t, \tau_{0}, \bar{\phi}\right)\right|<1$ for all $t \geq \tau_{0}$. Changing the order of integration (15) gives,

$$
\begin{aligned}
\left|\int_{t_{0}}^{\tau_{0}}\left(\int_{\tau_{0}}^{t} R(t, \sigma(s)) B(s, u) \Delta s\right) \bar{\phi}(u) \Delta u\right| & \leq\left|x\left(t, \tau_{0}, \bar{\phi}\right)\right|+\left|R\left(t, \tau_{0}\right)\right|\|\bar{\phi}\| \\
& \leq 3 .
\end{aligned}
$$

That is,

$$
\left.\mid \int_{t_{0}}^{\tau_{0}}\left(\int_{\tau_{0}}^{t} R(t, \sigma(s)) B(s, u) \Delta s\right) \phi(u) \Delta u\right) \mid \leq \frac{3}{\delta} .
$$

This implies that

$$
\sup _{t \in\left[t_{0}, \infty\right)_{\mathbb{T}}}\left\{\left|R\left(t, \tau_{0}\right)\right|+\int_{t_{0}}^{\tau_{0}}\left|\int_{\tau_{0}}^{t} R(t, \sigma(s)) B(s, u) \Delta s\right| \Delta u\right\} \leq \frac{5}{\delta} .
$$

Lemma 4.3. Let $R(t, s)$ be the solution of (14). Then there exists a constant $K$ such that $|R(t, s)| \leq K$ for $t \geq s \geq t_{0}$ if and only if

$$
\sup _{t \in\left[t_{0}, \infty\right)_{\mathbb{T}}}\left|\int_{s}^{t} R(t, \sigma(u))\left(A(u)+\int_{\sigma(s)}^{u} B(u, v) \Delta v\right) \Delta u\right|<+\infty .
$$

Proof. Integrating (14) from $s$ to $t$ yields

$$
R(t, s)=I+\int_{s}^{t} R(t, \sigma(u)) A(u) d u+\int_{s}^{t} \int_{v}^{t} R(t, \sigma(u)) B(u, v) \Delta u \Delta v .
$$

Interchange the order of integration in the last term to obtain

$$
\begin{aligned}
R(t, s) & =I+\int_{s}^{t} R(t, \sigma(u)) A(u) \Delta u+\int_{s}^{t} R(t, u) \int_{\sigma(s)}^{u} B(u, v) \Delta u \Delta v \\
& =I+\int_{s}^{t} R(t, \sigma(u))\left(A(u)+\int_{\sigma(s)}^{u} B(u, v) \Delta v\right) \Delta u .
\end{aligned}
$$

This implies the assertion of the lemma. 
Using Lemma 4.3, we obtain the following theorem which is more practical when the integrals of $R(t, s)$ with $A(t)$ and $\int_{t_{0}}^{t} B(t, s) \Delta s$ can be estimated.

Theorem 4.4. The zero solution of (5) is uniformly stable if and only if (26) holds and

$$
\sup _{t \in\left[t_{0}, \infty\right)_{\mathbb{T}}} \int_{t_{0}}^{\tau_{0}}\left|\int_{\tau_{0}}^{t} R(t, \sigma(s)) B(s, u) \Delta s\right| \Delta u<+\infty .
$$

We end this section with the following corollary.

Corollary 4.5. Suppose that

$$
\sup _{t \in\left[\tau_{0}, \infty\right)_{\mathbb{T}}} \int_{t_{0}}^{t}|R(t, \sigma(s))|\left(|A(s)|+\int_{t_{0}}^{s}|B(s, u)| \Delta u\right) \Delta s<+\infty .
$$

Then there exists a constant $K$ such that $|R(t, s)| \leq K$ for $t \geq s \geq t_{0}$ and the zero solution of (5) is US.

\section{Application to Scalar Equations}

In this section we apply the result of Theorem 4.3 and prove necessary and sufficient conditions for the uniform stability of the zero solution of the integrodynamic scalar equation

$$
x^{\Delta}(t)=a(t) x(t)+\int_{t_{0}}^{t} b(t, s) x(s) \Delta s, \quad t \in\left[t_{0}, \infty\right)_{\mathbb{T}} .
$$

It is worth mentioning that in [5], the authors used the notion of Lyapunov functionals and studied the boundedness of solutions of nonlinear forms of (28). First we will deal with the $\Delta$ - derivative of $|x(t)|$. In the continuous case one can easily find

$$
\frac{d}{d t}|x(t)|=\frac{x(t)}{|x(t)|} x^{\prime}(t)
$$

by using the equation $x^{2}(t)=|x(t)|^{2}$ and the product rule in real case, we have

$$
|x|^{\Delta}=\frac{x+x^{\sigma}}{|x|+\left|x^{\sigma}\right|} x^{\Delta} \text { for } x \neq 0,
$$

since the product rule is changed to $(f g)^{\Delta}=f^{\Delta} g^{\sigma}+f g^{\Delta}$ in time scale calculus. That is, the coefficient of $x^{\Delta}$ in (29) depends not only on the sign of $x(t)$ but 
also on that of $x^{\sigma}(t)$. Therefore, the equality $|x|^{\Delta}=\frac{x}{|x|} x^{\Delta}$ holds only if $x x^{\sigma} \geq 0$ and $x \neq 0$. Let us keep this case distinct from the case $x x^{\sigma}<0$ by separating the time scale $\mathbb{T}$ into two parts as follows

$$
\begin{aligned}
& \mathbb{T}_{-}:=\left\{s \in \mathbb{T}: x(s) x^{\sigma}(s)<0\right\} \\
& \mathbb{T}_{+}:=\left\{s \in \mathbb{T}: x(s) x^{\sigma}(s) \geq 0\right\}
\end{aligned}
$$

Note that the set $\mathbb{T}_{-}$consists only of right scattered points of $\mathbb{T}$. To see the relation between $|x|^{\Delta}$ and $\frac{x}{|x|} x^{\Delta}$ we prove the next result. For a proof of the next lemma we refer to our study [3].

Lemma 5.1. Let $x \neq 0$ be $\Delta$-differentiable. Then

$$
|x|^{\Delta}=\frac{x}{|x|} x^{\Delta} \text { for } t \in \mathbb{T}_{+}
$$

and

$$
\frac{x}{|x|} x^{\Delta} \leq|x|^{\Delta} \leq-\frac{x}{|x|} x^{\Delta} \text { for } t \in \mathbb{T}_{-}
$$

Theorem 5.2. Suppose that $a(t)$ does not change sign. Then the zero solution of (28) is uniformly stable if and only if there exist a constant $K$ such that

$$
a(t)+K \int_{t_{0}}^{\sigma(t)}|b(t, s)| \Delta s \leq 0
$$

and

$$
\min \left\{a(s)\left(-1+\frac{1}{K}\right), a(s)\left(1+\frac{1}{K}\right)\right\}>0
$$

Proof. Let

$$
V(s)=|R(t, s)|+\int_{s}^{t} \int_{t_{0}}^{s}|R(t, \sigma(u))||b(u, v)| \Delta v \Delta u .
$$

Then, by Lemma 5.1 we have 


$$
\begin{aligned}
V^{\Delta_{s}}(s) & =|R(t, s)|^{\Delta_{s}}-\int_{0}^{\sigma(s)}|R(t, \sigma(s))||b(s, v)| \Delta v \\
& +\int_{s}^{t}|R(t, \sigma(u))||b(u, s)| \Delta u \\
& \geq \frac{R(t, s)}{|R(t, s)|} R^{\Delta_{s}}(t, s)-\int_{0}^{\sigma(s)}|R(t, \sigma(s))||b(s, v)| \Delta v \\
& +\int_{s}^{t}|R(t, \sigma(u))||b(u, s)| \Delta u \\
& \geq-a(s) \frac{R(t, s)}{|R(t, s)|} R(t, \sigma(s))-\int_{\sigma(s)}^{t}|R(t, \sigma(u))||b(u, s)| \Delta u \\
& -\int_{0}^{\sigma(s)}|R(t, \sigma(s))||b(s, v)| \Delta v+\int_{s}^{t}|R(t, \sigma(u))||b(u, s)| \Delta u \\
& \geq-a(s) \frac{R(t, s)}{|R(t, s)|} R(t, \sigma(s))-\int_{0}^{\sigma(s)}|b(s, v)| \Delta v|R(t, \sigma(s))|
\end{aligned}
$$

where we have used

$$
\int_{s}^{t}|R(t, \sigma(u))||b(u, s)| \Delta u \geq \int_{\sigma(s)}^{t}|R(t, \sigma(u))||b(u, s)| \Delta u .
$$

If $R(t, s) R(t, \sigma(s)) \geq 0$, then $R(t, s) R(t, \sigma(s))=|R(t, s)||R(t, \sigma(s))|$. As a consequence, we have from (33) that

$$
\begin{aligned}
V^{\Delta_{s}}(s) & \geq\left(-a(s)-\int_{t_{0}}^{\sigma(s)}|b(u, v)| \Delta v\right)|R(t, \sigma(s))| \\
& \geq a(s)\left(-1+\frac{1}{K}\right)|R(t, \sigma(s))|,
\end{aligned}
$$

where we have used (31).

Also, if $R(t, s) R(t, \sigma(s)) \leq 0$, then $R(t, s) R(t, \sigma(s))=-|R(t, s)||R(t, \sigma(s))|$. As a consequence, we have from (33) that

$$
\begin{aligned}
V^{\Delta_{s}}(s) & \geq\left(a(s)-\int_{0}^{\sigma(s)}|b(u, v)| \Delta v\right)|R(t, \sigma(s))| \\
& \geq a(s)\left(1+\frac{1}{K}\right)|R(t, \sigma(s))|,
\end{aligned}
$$


where we have used (31), again.

Thus, (34) and (35) imply that

$$
V^{\Delta_{s}}(s) \geq \gamma(s)|R(t, \sigma(s))|
$$

where

$$
\gamma(s):=\min \left\{a(s)\left(-1+\frac{1}{K}\right), a(s)\left(1+\frac{1}{K}\right)\right\} .
$$

This along with (32) yields that for any $\tau_{0}$ with $t \geq \tau_{0}, V\left(\tau_{0}\right) \leq V(t)=$ $|R(t, t)|=1$. Thus, (20) is satisfied since

$$
\left|R\left(t, \tau_{0}\right)\right|+\int_{\tau_{0}}^{t} \int_{t_{0}}^{\tau_{0}}|R(t, \sigma(u))||b(u, v)| \Delta v \Delta u=V\left(\tau_{0}\right) \leq 1 .
$$

Example 5.3. Let $\mathbb{T}=\{0,1,2 \ldots\}$. Consider the scalar equation

$$
x(t+1)=\frac{x(t)}{2}-\sum_{s=0}^{t} \frac{x(s)}{(s+1)(s+2)}, \quad t \in\{0,1,2 \ldots\},
$$

which can be expressed in the form of Eq. (28) as follows

$$
\Delta x(t)=a(t) x(t)+\sum_{s=0}^{t-1} b(t, s) x(s)
$$

where $\Delta x(t):=x(t+1)-x(t)$,

$$
a(t)=-\frac{1}{2}-\frac{1}{(t+1)(t+2)}, \text { and } b(t, s)=-\frac{1}{(s+1)(s+2)} .
$$

If we let $K=-1 / 2$, then we have

$$
a(t)+K \int_{t_{0}}^{\sigma(t)}|b(t, s)| \Delta s=-1-\frac{1}{(t+1)(t+2)}+\frac{1}{2(t+1)}<0
$$

for $t \in\{0,1,2 \ldots\}$ and

$$
\min _{t \in \mathbb{T}}\left\{a(t)\left(-1+\frac{1}{K}\right), a(t)\left(1+\frac{1}{K}\right)\right\}=\frac{1}{2}+\frac{1}{(t+1)(t+2)}>0 .
$$

This means that the conditions (31) and (32) hold and the zero solution of (36) is uniformly stable by Theorem 5.2. 
Remark 5.4. In [9, Example 3.1], the authors ask for the existence of $K \in$ $(0,1)$ such that

$$
-\widetilde{a}(t)+K\left[1-\sum_{s=0}^{t}|b(t, s)|\right]>0
$$

in order to show that $\Delta V(s):=V(s+1)-V(s)>0$, which is needed for uniform stability of the zero solution of

$$
x(t+1)=\widetilde{a}(t) x(t)+\sum_{s=0}^{t} b(t, s) x(s) .
$$

In Example 5.3 we obtained uniform stability of the zero solution of (36) even though there is no such $K \in(0,1)$ such that condition (37) holds. To see this we let $\widetilde{a}(t)=1 / 2$ and $b(t, s)=-\frac{1}{(s+1)(s+2)}$. Then

$$
-\widetilde{a}(t)+K\left[1-\sum_{s=0}^{t}|b(t, s)|\right]=-\frac{1}{2}+\frac{K}{t+2}<0
$$

whenever $K \in(0,1)$ and $t \in\{0,1,2 \ldots\}$.

Remark 5.5. In [8, Example 2.3], the authors considered the scalar equation

$$
x^{\prime}(t)=a(t) x(t)+\int_{0}^{t} b(t, s) x(s) d s
$$

and the Lyapunov functional

$$
V(s)=|R(t, s)|+\int_{s}^{t} \int_{0}^{s}|R(t, u)||b(u, v)| d v d u .
$$

In order to have

$$
\liminf _{h \rightarrow 0^{+}} \frac{1}{h}[V(t+h)-V(t)] \geq 0
$$

they assumed the existence of a constant $K>1$ such that for $t \geq 0$

$$
a(t)+K \int_{0}^{t}|b(t, s)| d s \leq 0
$$

The above condition is needed to show uniform stability. We observe that our conditions (31) and (32) allow $K$ to be negative. For example if we assume $a<0$ and take $K=-\frac{1}{2}$, then

$$
\min \left\{a(s)\left(-1+\frac{1}{K}\right), a(s)\left(1+\frac{1}{K}\right)\right\}=\min \{-a(s),-3 a(s)\}=-a(s)>0 .
$$

Therefore, condition (31) is satisfied for any function $b(t, s)$. 
In [9, Example 3.1] and [8, Example 2.3], the authors also ask for more conditions to obtain uniform asymptotic stability. However, the conditions that we compared to our results were necessary only for uniform stability. If the authors in [9, Example 3.1] and [8, Example 2.3] only required the above mentioned conditions (i.e., (37) and (38)), then uniform stability would have been deduced. Thus, this paper improved the results of [9] and [8]. Also, one can easily specializes the results of this paper to $q$-difference equations.

\section{References}

[1] Adivar, M., Principal matrix solutions and variation of parameters for Volterra integro-dynamic equations on time scales, Glasg. Math. J. 53 (2011) 463-480.

[2] Adıvar, M. and Raffoul, Y. N., Existence of resolvent for Volterra integral equations on time scales, Bull. of Aust. Math. Soc., 82(1), (2010), 139155 .

[3] Adıvar, M. and Raffoul, Y. N., Existence results for periodic solutions of integro-dynamic equations on time scales, Ann. Mat. Pura Appl., 188 (4), 2009, 543-559.

[4] Adıvar, M., Function bounds for solutions of Volterra integro-dynamic equations on time scales, Electron. J. Qual. Theory Differ. Equ., No. 7. (2010), pp. 1-22.

[5] Akın-Bohner, E. and Raffoul Y. N., Boundedness in functional dynamic equations on time scales, Adv. Difference Equ., Volume 2006, Art. ID 79689, Pages 1-18.

[6] Bohner, M. and Peterson, A., Dynamic Equations on Time Scales, An introduction with applications. Birkhäuser Boston Inc., Boston, MA, 2001.

[7] Bohner, M. and Peterson, A., Advances in Dynamic Equations on Time Scales, Birkhäuser Boston Inc., Boston, MA, 2003.

[8] Eloe, P., Islam, M., and Zhang, B., Uniform asymptotic stability in linear Volterra integro-differential equations with applications to delay systems, Dynam. Systems Appl., 9 (2000), 331-344.

[9] Khandaker T. M., Raffoul Y. N, Stability properties of linear Volterra discrete systems with nonlinear perturbation, J. Difference Equ. Appl. 8 (2002), no. 10, 857-874. 
[10] Kulik, T. and Tisdell, C. C., Volterra integral equations on time scales: Basic qualitative and quantitative results with applications to initial value problems on unbounded domains. Int. J. Difference Equ. 3 (2008), no. 1, 103-133.

[11] Miller, R. K., Asymptotic stability properties of Volterra integrodifferential systems, J. Differential Equations, 10(1971), 485-506

[12] Miller, R. K., Nonlinear Volterra integral equations. W. A. Benjamin, Inc., Menlo Park, Calif., 1971.

[13] Tisdell, C. C. and Zaidi, A., Basic qualitative and quantitative results for solutions to nonlinear, dynamic equations on time scales with an application to economic modelling. Nonlinear Anal., Vol. 68(2008), No. 11,. $3504-3524$.

Murat Advar,

Department of Mathematics,

Izmir University of Economics,

Sakarya Cad. No: 156, 35330 Balova, zmir, Turkey.

Email: murat.adivar@ieu.edu.tr

Youssef N. Raffoul,

Department of Mathematics,

University of Dayton,

Dayton, OH 45469-2316 USA.

Email: youssef.raffoul@notes.udayton.edu 